\title{
PI3K/Akt inhibitor partly decreases TNF-a- induced activation of fibroblast-like synoviocytes in osteoarthritis
}

\author{
Songyang Liu ${ }^{1 \dagger}$, Chenxi Cao ${ }^{1,2 \dagger}$, Yujun Zhang ${ }^{3}$, Guangyu Liu', Weixia Ren ${ }^{3}$, Yanqi Ye ${ }^{1}$ and Tiezheng Sun ${ }^{*}$ (D)
}

\begin{abstract}
Background: The Cadherin-11 and PI3K/Akt pathway are increasingly recognized as the potential therapeutic target of osteoarthritis (OA) synovitis. The study aimed to investigate the role of PI3K/Akt signaling pathway in the expression of Cadherin-11 and migration and invasive capacity of fibroblast-like synoviocytes (FLS) of OA patients under stimulation of TNF-a and to explore the effect of the PI3K/Akt inhibitor and Cadherin-11 antibody in the therapy of the collagenase-induced osteoarthritis (CIOA) mice.

Methods: FLS were primarily cultured from synovium of osteoarthritic patients during total knee arthroplasty. Under the simulation of TNF-a, with or without PI3K/Akt inhibitor LY294002, Cadherin-11 expression was detected by real-time PCR and Western blot, as well as the migration and invasive capacity changes of OA FLS. Cadherin-11 antibody was injected intraarticularly or LY294002 was injected intraperitoneally in CIOA mice to evaluate the changes of synovitis score, cartilage damage, and Cadherin-11 expression.

Results: TNF-a stimulation increased Cadherin-11 expression at mRNA and protein level in OA FLS and also increased the phosphorylation-dependent activation of Akt. PI3K inhibitor LY294002 attenuated TNF-a-induced overexpression of Cadherin-11 and decreased the invasive capacity of OA FLS. Intraperitoneal injection of PI3K inhibitor LY294002 could decrease the Cadherin-11 protein expression in synovium of CIOA mice, although it has no significant inhibitory effect on synovitis and cartilage damage. Intraarticular injection of Cadherin-11 antibody attenuated the synovitis and cartilage damage in the CIOA joints and decreased Cadherin-11 expression in the synovial lining.
\end{abstract}

Conclusions: PI3K/Akt pathway was associated with TNF-a-induced activation of OA FLS, which may involve in the pathogenesis of osteoarthritis. Anti-Cadherin-11 therapy in CIOA mice could attenuate the pathological changes of OA joints.

Keywords: PI3K/Akt inhibitor, TNF-a, Cadherin-11, Osteoarthritis, Fibroblast-like synoviocytes

\section{Background}

Osteoarthritis (OA) is the most common form of arthritis and forms a major burden to health care $[1,2]$. Previously, we have long considered it as a non-inflammatory degenerative joint disease, characterized by articular cartilage degradation. However, in recent years, many research papers indicate that osteoarthritis contains pathological

\footnotetext{
* Correspondence: tiezheng_sun1973@163.com

+Songyang Liu and Chenxi Cao contributed equally to this study.

${ }^{1}$ Arthritis Clinic and Research Center, People's Hospital, Peking University, Beijing 100044, People's Republic of China

Full list of author information is available at the end of the article
}

changes of all structures within the joint, including the synovial inflammation, hyaline articular cartilage loss, and bony remodeling changes [3]. Synovial inflammation is increasingly recognized as contributing to the pathology during progression of osteoarthritis [4]. An arthroscopic study also suggests that synovitis occurs in $50 \%$ of patients with OA [5], and the synovial activation was associated with cartilage pathology [6]. Previous researches suggest that many inflammation cytokines, such as TNF- $\alpha$ and IL$1 \beta$, might participate in OA synovial inflammation $[7,8]$. It has been assumed that inflammatory cytokines might

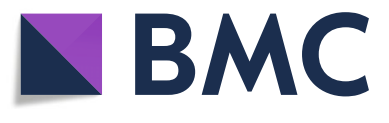

(c) The Author(s). 2019 Open Access This article is distributed under the terms of the Creative Commons Attribution 4.0 International License (http://creativecommons.org/licenses/by/4.0/), which permits unrestricted use, distribution, and reproduction in any medium, provided you give appropriate credit to the original author(s) and the source, provide a link to the Creative Commons license, and indicate if changes were made. The Creative Commons Public Domain Dedication waiver (http://creativecommons.org/publicdomain/zero/1.0/) applies to the data made available in this article, unless otherwise stated. 
act as triggers of the inflammatory cascade amplification in local synovitis in OA.

It has been proposed that fibroblast-like synoviocytes (FLS) are actively involved in chronic inflammatory reactions. The molecular mechanisms of their sustained activation might be contributed to inflammatory cascades. Cadherin-11 is a classical molecule that mediates hemophilic cell-to-cell adhesion in FLS, plays an important role in the development of the normal synovium lining layer of the joint [9-11], and has been identified to participate in the cartilage invasion except for its adhesive function [10]. Cadherin-11-deficient mice have a hypoplastic synovial lining, display disorganized synovial reaction to inflammation, and are resistant to inflammatory arthritis [12]. Our previous study demonstrated that Cadherin-11 expression in FLS was positively related to the degree of synovitis [13]. As a predominant inflammatory factor, TNF- $\alpha$ plays a vital role in the upregulation of Cadherin-11 in FLS. However, the underlying mechanism for the upregulation of Cadherin-11 expression in FLS by the stimulation of TNF- $\alpha$ was rarely investigated.

The study objective was to explore whether PI3K/Akt signaling pathway was involved in the TNF- $\alpha$-induced upregulation of Cadherin-11, as well as the migration and invasive capacity of OA FLS. In the meantime, we aimed to examine the potential therapeutic effect with PI3K/Akt inhibitor and anti-Cadherin-11 antibody in collagenase-induced osteoarthritis (CIOA) mice.

\section{Materials and methods}

\section{Isolation and culture of fibroblast-like synoviocytes}

Synovial tissues were collected at the time of total knee arthroplasty (TKA) from 26 patients with primary OA in the Arthritis Clinic and Research Center, People's Hospital, Peking University, Beijing, China. The diagnosis of OA fulfilled the criteria of the American College of Rheumatology (ACR) in 1985 [12]. The mean age of the osteoarthritic patients was 65.8 years old (range 55-76), 5 males and 21 females.

FLS were isolated by enzymatic dispersion of synovial tissue from the OA patients [14]. Briefly, synoviocyte suspensions were prepared from the synovial membranes after mincing and incubation with $1 \mathrm{mg} / \mathrm{ml}$ type I collagenase (Invitrogen, CA, USA) in low-glucose Dulbecco's modified Eagle's medium (DMEM) for $2-3 \mathrm{~h}$ at $37^{\circ} \mathrm{C}$. The cell suspensions were filtered through a 70$\mu \mathrm{m}$ cell strainer (BD Biosciences, CA, USA), extensively washed, and placed in tissue culture dishes in DMEM supplemented with $10 \%$ (volume/volume, v/v) fetal bovine serum (FBS, Invitrogen, Australia), $100 \mathrm{U} / \mathrm{ml}$ penicillin, and $100 \mu \mathrm{g} / \mathrm{ml}$ streptomycin in a $37^{\circ} \mathrm{C}, 5 \% \mathrm{CO}_{2}$ incubator.

After the third passage, non-FLS cells completely disappeared from these culture systems, and the remaining cells were mainly FLS as previously described [15], which were proved by flow cytometry. FLS from the third to the sixth passages were chosen for the subsequent experiments.

Isolated FLS were seeded in six-well plates containing $3 \mathrm{ml}$ DMEM supplemented with 10\% FBS and grown to subconfluence of $80 \%$. After starvation in the serum-free culture medium for $24 \mathrm{~h}$, the regular medium was replaced and FLS were treated with TNF- $\alpha$ and/or PI3K/ Akt inhibitor. For TNF- $\alpha$ treatment, each culture plate was treated by the recombinant human TNF- $\alpha$ (R\&D Systems, Minneapolis, MN, USA) for $24 \mathrm{~h}$ at concentrations of $10 \mathrm{ng} / \mathrm{ml}$. Control cultures containing no cytokines were grown in parallel. To evaluate the effect of PI3K/Akt on the FLS activation induced by TNF- $\alpha$, FLS were pre-incubated with $10 \mu \mathrm{M}$ LY2940002 (PI3K/Akt inhibitor) for $1 \mathrm{~h}$ before TNF- $\alpha$ stimulation. The inhibitor was diluted in dimethyl sulfoxide (DMSO), and control cells were pre-incubated with equivalent amounts of DMSO alone.

\section{Quantitative real-time PCR}

Total ribonucleic acid (RNA) was obtained from OA FLS using TRIzol reagent (Invitrogen, Camarillo, USA) according to the instructions. After determining the spectral value with NanoVue Plus spectrophotometer (GE Healthcare, Piscataway, USA), $0.1 \mu \mathrm{g}$ RNA was reversely transcribed into cDNA using SuperScript III First-Strand Synthesis System (Invitrogen).

Subsequently, qPCR was performed using MiniOpticon qPCR (Bio-Rad, Hercules, USA). The reaction mixture contained $2 \mu \mathrm{l}$ cDNA, $6.4 \mu \mathrm{l}$ pure water, $0.8 \mu \mathrm{l}$ forward and reverse primer, and $10 \mu \mathrm{l}$ SYBR qPCR Mix (QPS-201, Toyobo, Shanghai, China). The primer sequences for GAPDH were 5'-GTCTCCTCTGACTTCAACAGCG-3' (forward) and 5'-ACCACCCTGTTGCTGTAGCCAA-3' (reverse), and the primer sequences for Cadherin-11 were 5'-GATCGTCACACTGACCTCGACA-3' (forward) and 5'-CTTTGGCTTCCTGATGCCGATTG-3' (reverse).

The whole process consisted an initial activation step at $94{ }^{\circ} \mathrm{C}$ for $2 \mathrm{~min}$ followed by 40 amplification cycles: $94^{\circ} \mathrm{Cfor} 15 \mathrm{~s}$ (denaturation), $60^{\circ} \mathrm{C}$ for $20 \mathrm{~s}$ (renaturation), and $72{ }^{\circ} \mathrm{C}$ for $30 \mathrm{~s}$ (extension). The specificity of the primers was verified by the melting curve analysis. Data were analyzed using the comparative threshold method $(\Delta \Delta \mathrm{Ct})$, and the results were expressed as $2^{-\Delta \Delta \mathrm{Ct}}$.

\section{Western blot}

After treatment with TNF- $\alpha$ and PI3K/Akt inhibitor, total cell lysate was obtained by incubating the FLS monolayers with RIPA Kit (Solarbio, Peking, China). The protein concentration was determined using BCA protein assay kit (Solarbio, Peking, China). An identical amount of proteins $(20 \mu \mathrm{g})$ was separated using $8 \%$ SDS-PAGE and blotted onto PVDF membranes (Bio-Rad, Hercules, USA). Blots 
were blocked for $1 \mathrm{~h}$ with 5\% BSA in Tris-buffered saline (TBS) at room temperature and then incubated overnight at $4{ }^{\circ} \mathrm{C}$ with anti-Cadherin-11 (1:1500 dilution, Abcam, Cambridge, MA, USA), anti-Akt (1:2000 dilution), and anti-P-Akt (1:1000 dilution, Cell Signaling Technology, Beverly, MA, USA) antibodies. Subsequently, bound antibody was detected with horseradish peroxidase-labeled goat anti-rabbit IgG (1:1000 dilution, sc-2005, Santa Cruz, Dallas, USA) and visualized with an enhanced Chemiluminescence Kit (Thermo Scientific, Rockford, USA). Protein loading was checked and standardized by $\beta$-actin (anti- $\beta$-actin antibody, 1:2000 dilution; Proteintech, Chicago, USA). Quantification of the band intensity was performed using Image J software (National Institutes of Health, USA).

\section{Cell migration and invasion assay}

$2 \times 10^{4}$ OA FLS in serum-free DMEM medium were seeded in the upper chamber of the 8.0- $\mu \mathrm{m}$ pore filter migration inserts (Transwell; Corning, NY, USA) in 24-well cell culture plates, with or without TNF- $\alpha$. To evaluate the effect of PI3K/Akt on the migration and invasion capacity treated by TNF- $\alpha$, FLS were pre-incubated with $10 \mu \mathrm{M}$ LY294002 for $1 \mathrm{~h}$ before TNF- $\alpha$ stimulation for 24 $\mathrm{h}$. The lower chamber contained DMEM medium with $10 \%$ fetal bovine serum (FBS). After incubation for $24 \mathrm{~h}$, cells that had not penetrated the filter were removed from the top of the filter using cotton swabs, and the remaining cells were fixed in $4 \%$ (weight/volume, $\mathrm{w} / \mathrm{v}$ ) paraformaldehyde for $15 \mathrm{~min}$, then stained with $0.1 \%(\mathrm{w} / \mathrm{v})$ crystal violet, and counted. Values for migration were expressed as the average number of migrated cells bound per microscopic field $(\times 100)$. Three microscopic fields per membrane in triplicate experiments were counted.

For invasion assay, the migration inserts were coated with $20 \mu \mathrm{l}$ 10\% (v/v) Matrigel (BD Bioscience, San Diego, CA, USA) at $37^{\circ} \mathrm{C}$ for $45 \mathrm{~min}$. Then, $2 \times 10^{4} \mathrm{OA}$ FLS in serum-free DMEM medium were seeded likewise. After incubation for $24 \mathrm{~h}$, cells that had not penetrated the filter were removed from the top of the filter using cotton swabs, and the remaining cells were fixed in $4 \%(\mathrm{w} / \mathrm{v})$ paraformaldehyde for $15 \mathrm{~min}$, then stained with $0.1 \%(\mathrm{w} /$ v) crystal violet, and counted. Values for invasion were expressed as the average number of migrated cells on the underside of the filters per microscopic field $(\times 100)$. Three microscopic fields per membrane in triplicate experiments were counted.

\section{Induction of collagenase induced $\mathrm{OA}$ mice model}

Thirty male C57/BL6 mice aged 8-12 weeks were randomly divided into five groups with six for each group: (1) control group, (2) OA + isotype IgG group, (3) OA + Cadherin-11 antibody group, (4) OA+ LY2940002 (PI3K/Akt inhibitor) group, and (5) OA + DMSO group.
Experimental OA mice model was induced as previously described [16]. In brief, the procedure involves intraarticular administration of $10 \mu \mathrm{l}$ collagenase VII (containing 1 unit collagenase) (Sigma, St. Louis, MO) in the right knee on day 0 and day 2 .

After 2 weeks of collagenase induction, $2 \mu$ g isotype $\operatorname{IgG}$ (ZSGB-BIO, China, ZDR-5001) or Cadherin-11 antibody (sc-30,314, Santa-Cruz, USA) was separately intraarticularly administered twice a week for 2 weeks. Then, the mice were euthanized with $\mathrm{CO}_{2}$, and the right knees were isolated and processed for histological examination.

After 2 weeks of collagenase induction, $1 \mathrm{mg}$ LY294002 dissolved in $200 \mu \mathrm{l}$ 8\%DMSO diluted by PBS was inoculated intraperitoneally (i.p.) daily for 2 weeks. Two hundred microliters of $8 \%$ DMSO-PBS was inoculated i.p. daily for the control group. After 2 weeks, the mice were euthanized with $\mathrm{CO}_{2}$, and the right knees were isolated and processed for histological examination.

\section{Histological assessment of articular cartilage damage and synovitis score}

Intact mouse knees were dissected away from the skin, fixed in $4 \%$ paraformaldehyde for 2 days and decalcified in $10 \%$ formic acid for approximately 1 week. Then, the specimens were embedded in paraffin and sections $(4 \mu \mathrm{m})$ throughout the knee (five sections at $100 \mu \mathrm{m}$ distance) were required for the HE, Safranin O/Green staining, and Cadherin-11 staining.

Krenn's synovitis score on HE stained synovial membrane sections included the synovial hyperplasia graded by mean synovial lining layer thickness $(1-3$ scale, where $1=$ mean of $1-2$ cell layers, $2=$ mean of $3-5$ cell layers, and $3=$ mean of $>5$ cell layers), the density of stromal cells, and inflammatory infiltration of the sublining layer. This semiquantitative scoring method has been extensively validated.

Cartilage damage score was performed according to the OARSI scoring system on Safranin O/Green staining of knee joint sections, which was a modification from Chambers et al. [17]. The scoring system is recommended to apply to all four quadrants of the joint: medial tibial plateau (MTP), medial femoral condyle (MFC), lateral femoral condyle (LFC), and lateral tibial plateau (LTP). A score of 0 represents normal cartilage, $0.5=$ loss of PG with an intact surface, 1 = superficial fibrillation without loss of cartilage, $2=$ vertical clefts and loss of surface lamina (any \% or joint surface area), $3=$ vertical clefts/erosion to the calcified layer lesion for $1-25 \%$ of the quadrant width, $4=$ lesion reaches the calcified cartilage for $25-50 \%$ of the quadrant width, $5=$ lesion reaches the calcified cartilage for $50-75 \%$ of the quadrant width, and $6=$ lesion reaches the calcified cartilage for $>75 \%$ of the quadrant width. Five sections were obtained and evaluated for each mouse. The cartilage 
damage index was expressed as summed score which can be combined for the entire joint or split out for MTP, MFC, LTP, or LFC separately.

For immunohistochemical staining of Cadherin-11, paraffin sections $(4 \mu \mathrm{m})$ were routinely prepared, dewaxed with the endogenous peroxidase inactivated by $3 \%(\mathrm{v} / \mathrm{v})$ $\mathrm{H}_{2} \mathrm{O}_{2}$, then incubated in citrate buffer $(0.1 \mathrm{M}, \mathrm{pH} 6.0)$ at $98^{\circ} \mathrm{C}$ for about $20 \mathrm{~min}$. After blocking with $10 \%(\mathrm{v} / \mathrm{v})$ BSA, sections were incubated with 1:100 anti-Cadherin-11 rabbit polyclonal antibody (Clone WNTID1, Invitrogen, Camarillo, USA) overnight at $4{ }^{\circ} \mathrm{C}$. Parallel sections were incubated with a nonspecific isotope, and concentration matched antibody as negative control. After incubation with the primary antibody, sections were sequentially incubated with horseradish peroxidase-conjugate goat antirabbit immunoglobulin G (IgG) (Gene. Shanghai. China) for $60 \mathrm{~min}$ at room temperature and visualized with 3,3diaminobenzidine (Solarbio, Peking, China). Finally, the sections were counterstained with hematoxylin.

As described previously, Cadherin-11 staining was scored semiquantitatively based on intensity [categorized as 0 (absent), 1 (weak), 2 (moderate), or 3 (strong)] and the percentage of positively stained area [scored as $0(0-5 \%$ positive), 1 (6-25\%), 2 (26-50\%), $3(51-75 \%)$, or 4 (> 75\%)] in the lining layer and sublining layer [18]. The evaluator of the joints was blinded to the treatment groups.

\section{Statistical analysis}

The statistical analysis was performed using SPSS Version 20.0 (IBM Corp, NY, USA). The normally distributed data such as the values from Western blot and qRT-PCR were expressed as mean $\pm \mathrm{SD}$ and were analyzed using one-way analysis of variance (ANOVA). The abnormally distributed data like histological parameters of synovitis score and cartilage damage score of different groups were expressed as mean \pm SEM and compared by the Kruskal-Wallis non-parametric test. The MannWhitney $U$ test was used for direct comparisons between the two groups. $p$ values less than 0.05 were considered significant $\left(" p<0.05,{ }^{* * *} p<0.01,{ }^{* * * *} p<0.001\right)$.

\section{Results}

\section{Cadherin-11 upregulation by TNF-a was associated with} PI3K/Akt pathway

Cadherin-11 was increased at mRNA and protein level in OA FLS with TNF- $\alpha$ stimulation compared to the control group (Fig. 1a, b). Also, TNF- $\alpha$ stimulation could increase the phosphorylation-dependent activation of PI3K/Akt (Fig. 1b, d, $p<0.05$ ).

To explore the role of PI3K/Akt signaling pathway in expression of cadherin-11 induced by TNF- $\alpha$ in OA FLS, the specific PI3K inhibitor, LY294002, was used to pre-treat OA FLS before TNF- $\alpha$ stimulation. As shown in Fig. 1d, phosphorylation of Akt induced by TNF- $\alpha$ was significantly inhibited by LY294002 (Fig. 1d, $p<$ 0.001). Expression of Cadherin-11 protein induced by TNF- $\alpha$ was significantly attenuated after incubation with LY294002 (Fig. 1b, c, $p<0.05$ ). Increased expression of Cadherin-11 mRNA induced by TNF- $\alpha$ was slightly reduced by LY294002; however, it was not statistically significant (Fig. 1a, $p>0.05$ ).

PI3K inhibitor, LY294002, inhibits the migration and invasive capacity of OA FLS induced by TNF- $a$

As shown in Fig. 2, TNF- $\alpha$ stimulation enhanced the migration and invasive capacity of OA FLS. PI3K inhibitor, $10 \mu \mathrm{M}$ LY294002, inhibited in vitro invasion capacity of OA FLS stimulated by TNF- $\alpha$ (Fig. $2 \mathrm{~b}, p<0.05$ ), but only slightly inhibited the migration capacity of OA FLS (Fig. 2a, $p>0.05$ ). These results suggested that PI3K/Akt pathway might be involved in modification of the cellular invasiveness behavior of OA FLS.

\section{PI3K inhibitor and anti-Cadherin-11 therapeutics on synovial hyperplasia and cartilage damage in CIOA mice}

In the collagenase-induced OA mice, a clear thickening of the synovial lining layer was found compared to the control mice (Fig. 3a). As shown in Fig. 4a, digital image analysis showed that the cartilage damage mostly occurred in the medial tibial plateau (MTP) (Fig. 4b, $p<0.001$ ) and medial femoral condyle (MFC) (Fig. 4c, $p<0.01$ ).

After the treatment of LY294002, expression of Cadherin-11 in the synovial lining layer (Fig 3f, $p<0.01$ ) and sublining layer in CIOA was significantly reduced (Fig. 3g, $p<0.05$ ), but there was no significant change on synovial hyperplasia, sublining inflammatory infiltration, and density of stromal cells (Fig. 3b-d, $p>0.05$ ). Either the total synovitis score did not show any significant difference (Fig. 3e, $p>0.05$ ). The treatment of LY294002 showed no significant effect cartilage destruction in the four separate quadrants (Fig. $4 \mathrm{~b}-\mathrm{d}, p>0.05$ ) and the summed cartilage score (Fig. 4e, $p>0.05$ ) in the CIOA model.

In the Cadherin-11 antibody group, typical features of OA including synovial hyperplasia, sublining inflammatory infiltration, and density of stromal cells were significantly attenuated compared to the CIOA group (Fig. $5 \mathrm{~b}-$ $\mathrm{d}, p<0.05)$. The general synovitis score also decreased significantly after Cadherin-11 antibody treatment (Fig. 5e, $p<0.05)$. Expression of Cadherin-11 in the synovial lining layer in CIOA was reduced after treatment of Cadherin11 antibody (Fig. 5f, g, $p<0.001$ ). The cartilage damage score in MTP (Fig. 6b, $p<0.01$ ), MFC (Fig. 6c, $p<0.05$ ), and the summed score in the CIOA model was alleviated by injection of Cadherin-11 antibody (Fig. 6f, $p<0.01$ ).

\section{Discussion}

It has been proved that the PI3K/Akt signaling pathway can be activated following TNF- $\alpha$ exposure [19]. Feldman 


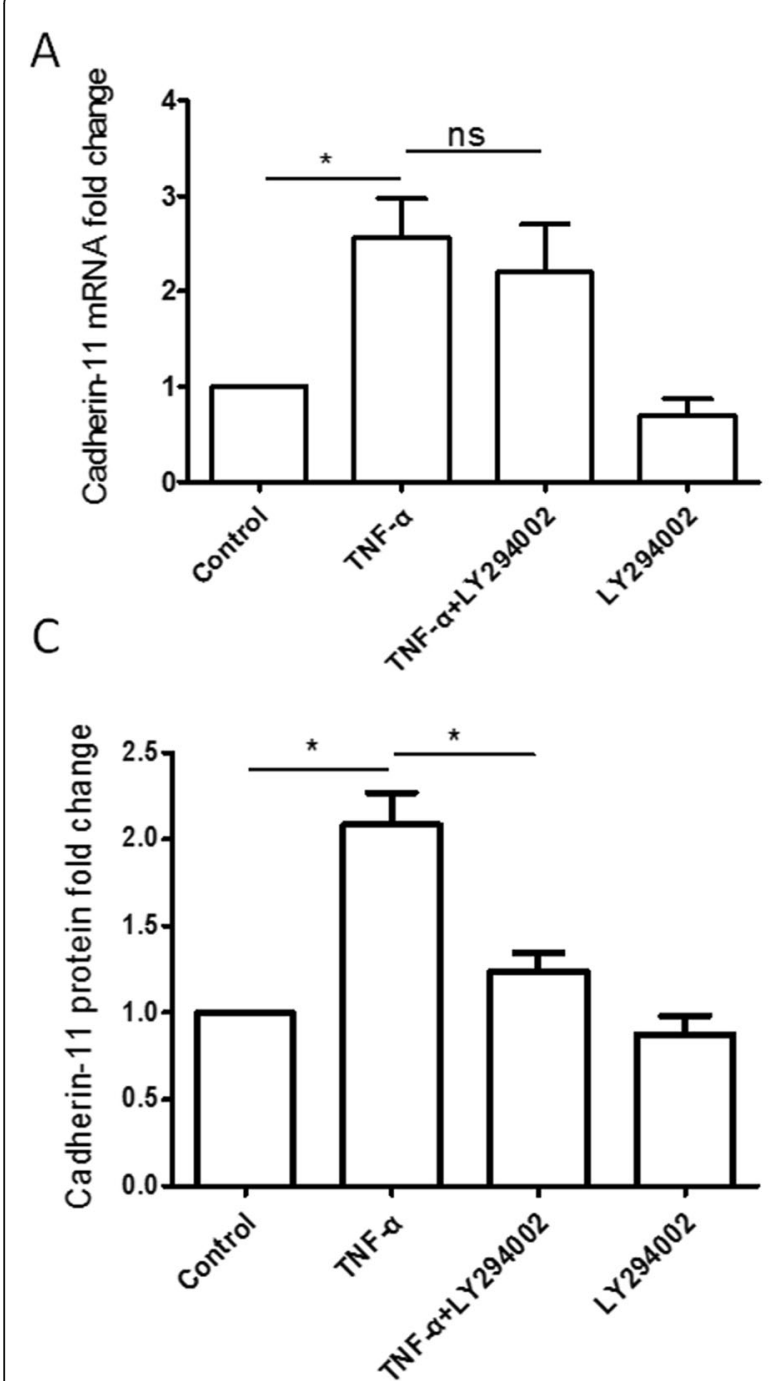

B

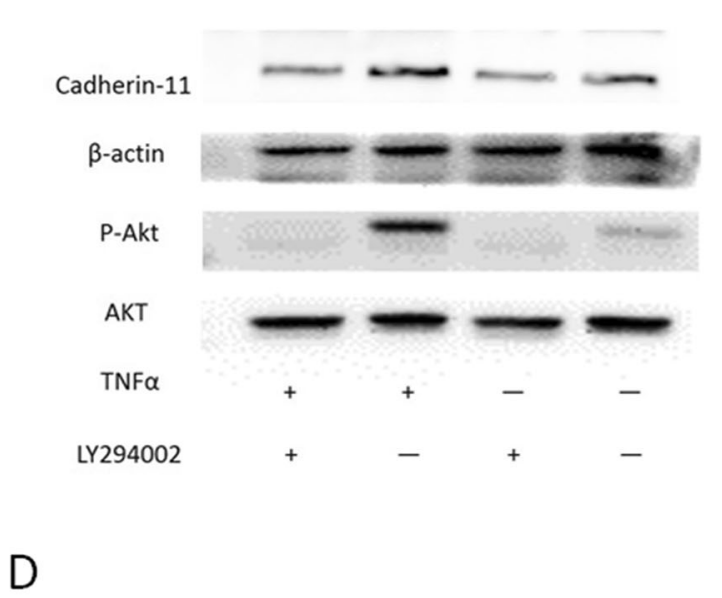

Fig. 1 Expression of Cadherin-11 and P-Akt in OA FLS induced by TNF-a with or without PI3K inhibitor LY294002. a Real-time PCR showed that cadherin-11 mRNA increases with the stimulation of TNF-a in OA FLS $(p<0.05)$, but PI3K inhibitor LY294002 had no significant inhibitory effect $(p>0.05)$. $\mathbf{b}$ Western blot showed that expression of Cadherin-11 and phosphorylation of Akt (P-Akt) increased with the stimulation of TNF-a in OA FLS ( $p<0.001)$, while PI3K inhibitor LY294002 had significant inhibitory effect $(p<0.001)$. c Quantification analysis of expression of Cadherin-11 protein. The amount of each protein was determined densitometrically and normalized to $\beta$-actin. $\mathbf{d}$ Quantification analysis of P-Akt. The amount of each protein was determined densitometrically and normalized to total Akt. The experiment was performed three times. Values were means \pm $\mathrm{SD},{ }^{*} p<0.05,{ }^{* *} p<0.01,{ }^{* * *} p<0.001$

et al. has also demonstrated that PI3K/Akt pathway can be activated by several cytokines such as TNF- $\alpha$ in RA synoviocytes [20,21]. In this study, the overexpression of Cadherin-11 in OA FLS after being treated with TNF- $\alpha$ was accompanied by the activation of P-Akt, which represents the persistent activation of PI3K/Akt pathway. However, when pre-treated with LY294002, the protein expression of P-Akt and Cadherin-11 in OA FLS was significantly reduced, although the inhibitory effects were not significant on Cadherin-11 mRNA level. In fact, from DNA to mRNA to protein expression, there are three levels of regulation including transcription, translation, and post-translation. Thus, the mRNA expression is only related to the transcription level, which cannot fully represent the whole process of regulation. Chang et al. [14] pointed out that the activation of Akt can cause the phosphorylation of GSK3 $\beta$ at the Ser9 site and thereby inhibiting its kinase activity. However, Vandooren et al. [15] reported that glycogen synthase kinase 3 beta (GSK-3 $\beta$ ) stabilizes Cadherin-11 mRNA expression by $\beta$-cateninindependent pathway in prostate cancer and breast cancer cells. It also regulates the stability of the untranslated region of Cadherin-11 at post-transcriptional level via $\beta$ catenin-dependent pathway, which leads to a decrease in 


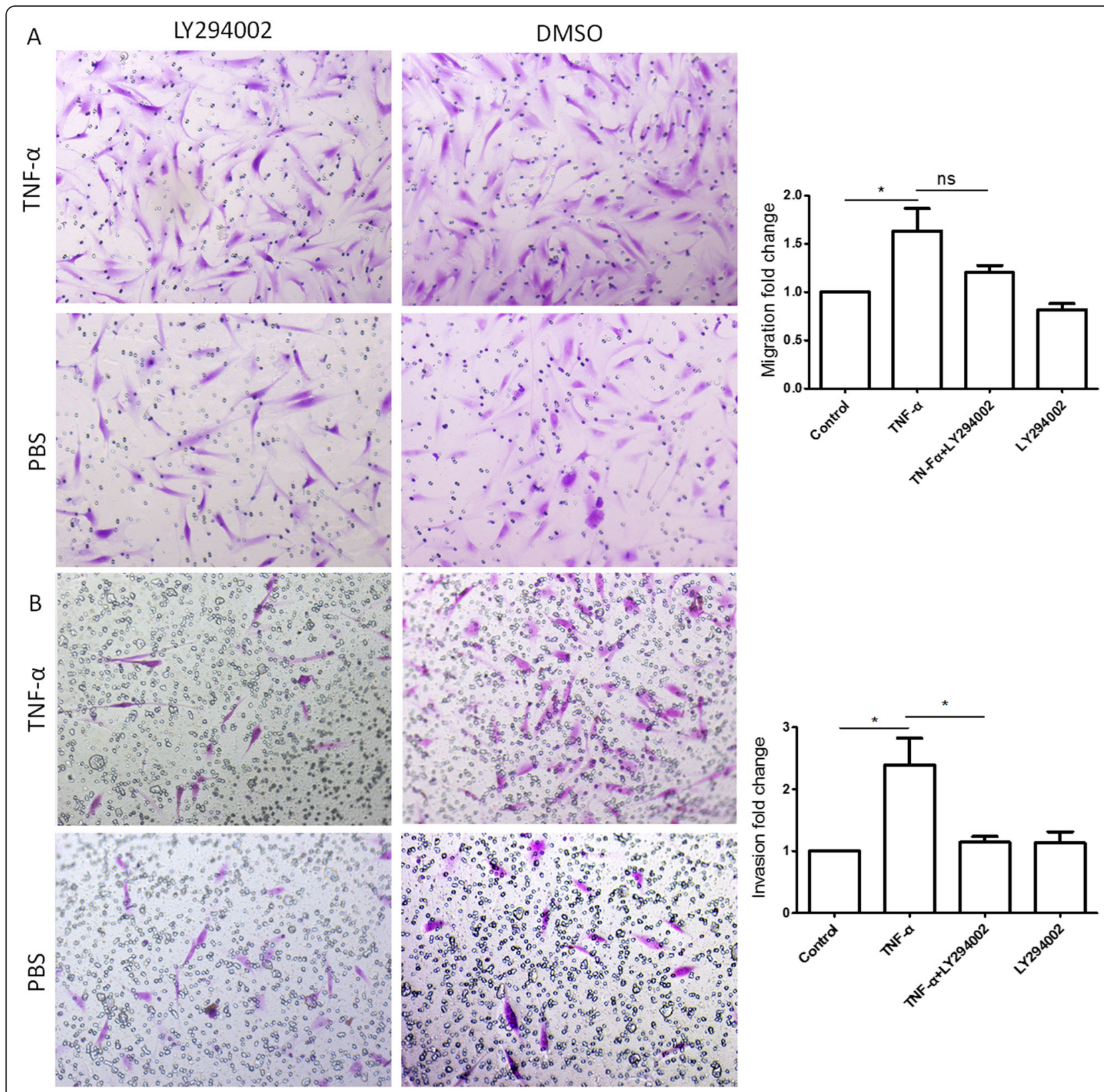

Fig. 2 Effect of LY294002 on the migration and invasive capacity of OA FLS with stimulation of TNF-a. TNF-a increased migration (a) and invasive (b) capacity of OA FLS $(p<0.05)$. PI3K inhibitor, LY294002, could inhibit the invasive capacity of OA FLS induced by TNF-a $(p<0.05)$, but has little inhibitory effect on migration of OA FLS $(p>0.05)$. Five fields per filter were selected randomly and photographed. All experiments were repeated three times. Data are expressed as mean \pm SD. ${ }^{*} p<0.05,{ }^{* *} p<0.01,{ }^{* *} p<0.001$

the expression level of Cadherin-11 protein. In combination with our results, the reason why Cadherin-11 did not show a significant decrease in mRNA level after treatment with LY294002 in OA FLS may be due to LY294002 causing the inhibition of Akt activation, which abates the inhibition of GSK-3 $\beta$ thus leading to the increased stability of Cadherin-11 mRNA, while the decrease of Cadherin-11 protein expression is caused by the regulation of transcriptional regulation of GSK3 $\beta$ through $\beta$-catenin-dependent pathway. Actually, the PI3K/Akt regulates a cascade of changes through its broad target proteins such as mTOR, NF-kB, GSK-3 $\beta$, and p53 [22], which suggests that PI3K might be involved in post-transcriptional regulation of cadherin-11. So, we concluded TNF- $\alpha$ induced the increases of Cadherin-11 expression via PI3K/Akt pathway in OA FLS, which was consistent with the fact that PI3K/ AKT pathway plays an important role in synovial inflammation [23]. 


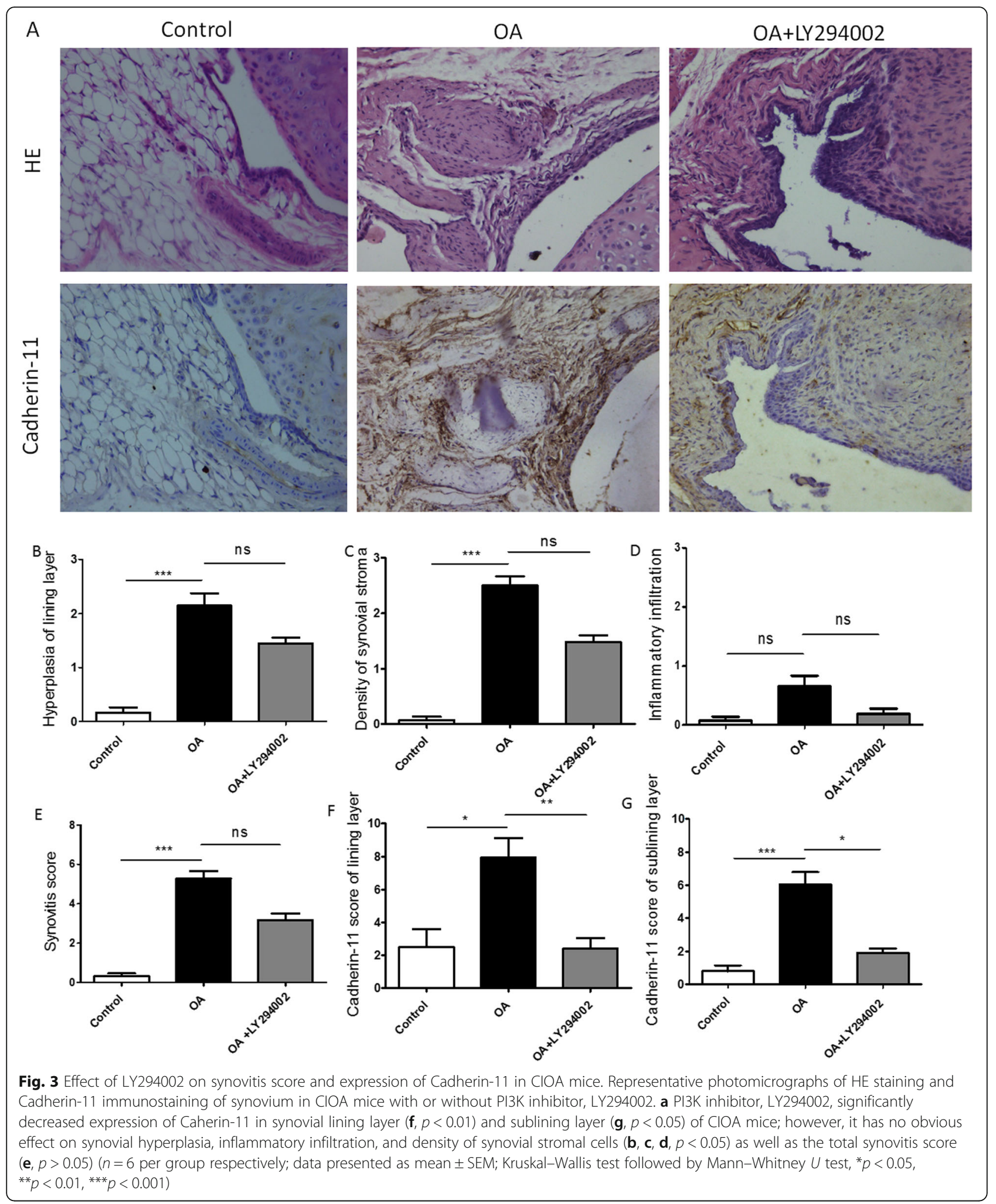

PI3K/Akt might serve as a cross-link between OA FLS invasion and cartilage damage. The migration of FLS to the cartilage and bone has been considered a critical step in the erosion of joints in OA and RA. It is well established that pro-inflammatory cytokines, such as IL- $1 \beta$ and TNF- $\alpha$, constitute key mediators of FLS migration and invasion [24]. We found that inhibition of PI3K/Akt could significantly decrease the invasive 


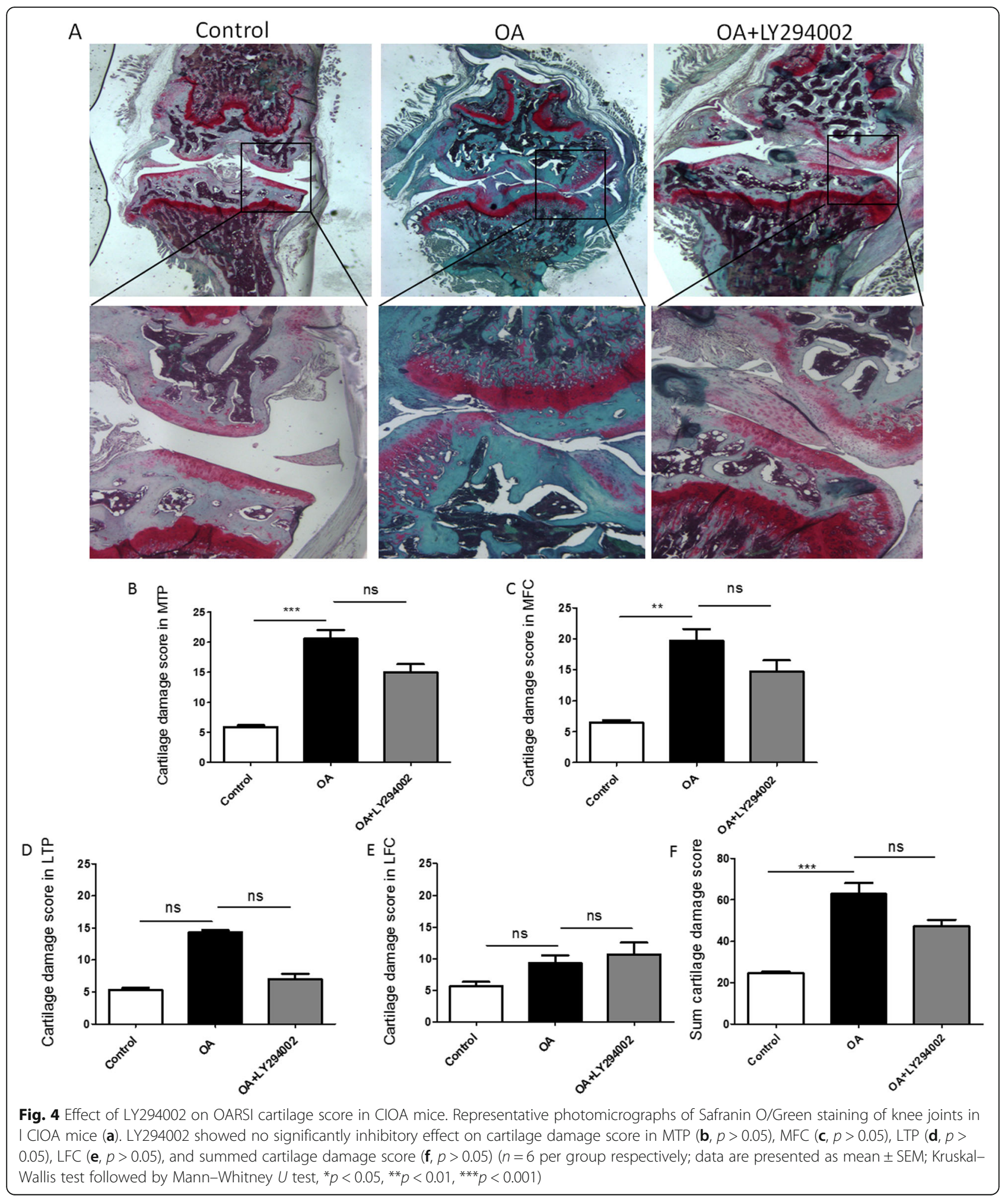

capacity of OA FLS under the stimulation of TNF- $\alpha$, but the inhibition of migration capacity was not significant. This implies that FLS following the inflammatory reaction might result in the invasion of FLS and destruction of cartilage. It is known that OA FLS was required to penetrate matrix in the invasion assay compared with the migration assay. Therefore, the reduced invasive ability may be related to the decreased 


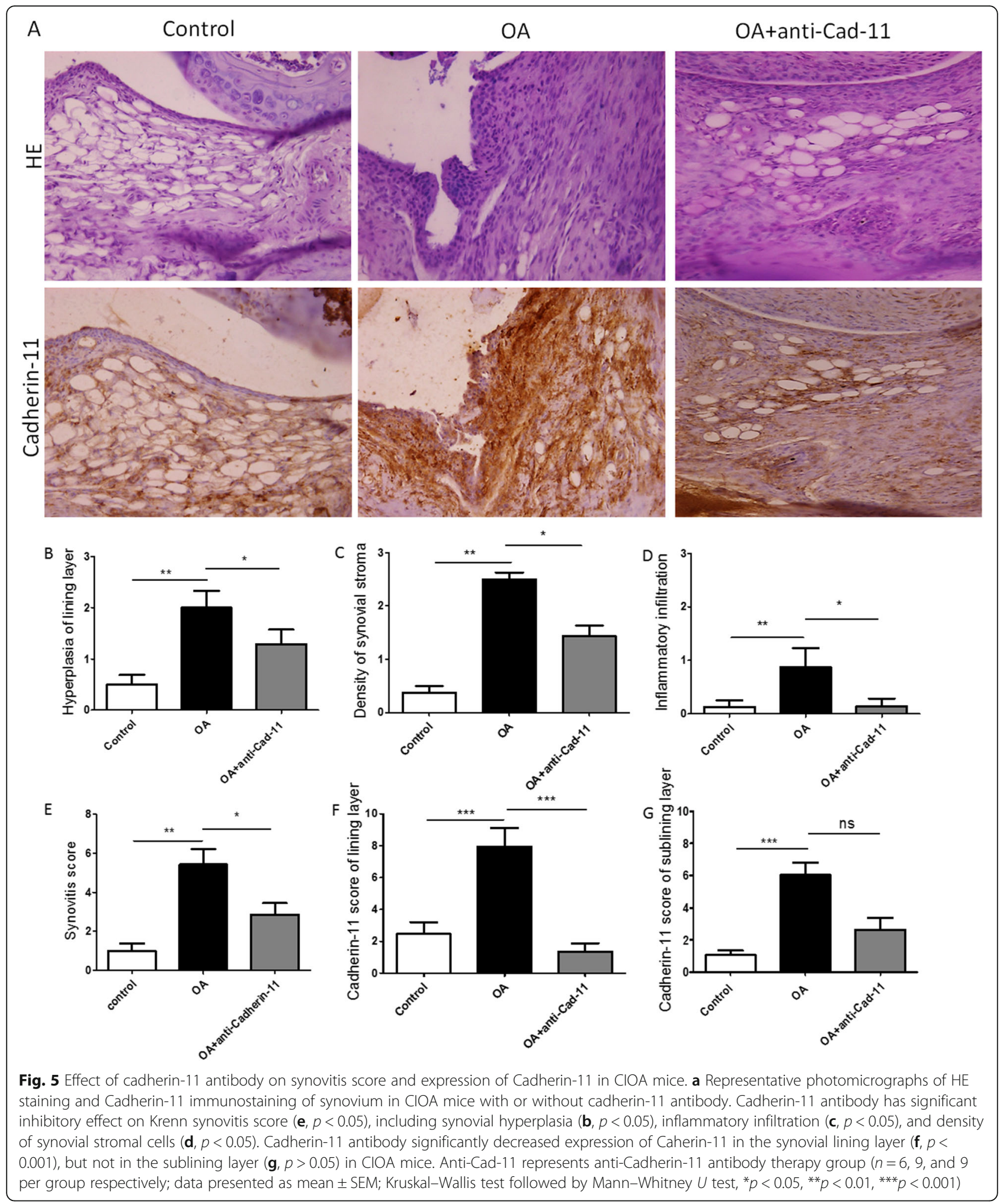

secretion of MMP3 or MMP9 after inhibition of PI3K/ Akt signaling. In addition to its role in FLS, PI3K/Akt activation can induce a series of downstream signaling pathways and target protein, including NF-kB, GSK-
$3 \beta$, and p53. $[25,26]$. confirmed that the activation of P-Akt and NF-kB signaling pathways plays an important role in the inflammatory factor-mediated secretion of MMPs. And these may explain why only the 


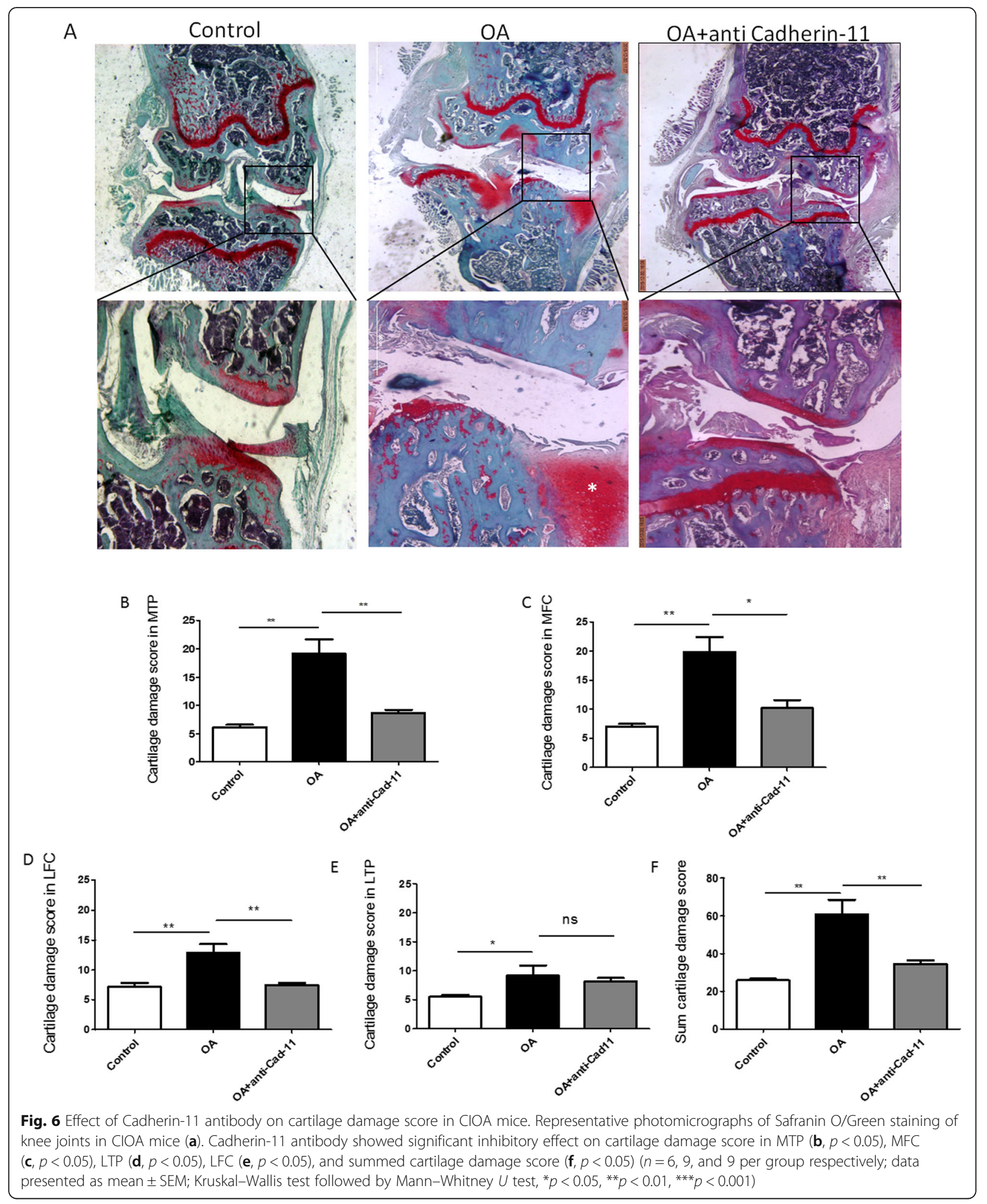


invasion capacity has changed while migration capacity has not.

Given the important roles uncovered for PI3K in promoting inflammation, many studies have investigated the effects of pharmacological inhibition of PI3K isoforms in animal models of inflammatory joint disease. The PI3K $\gamma$ inhibitors AS605240 and TASP0415914 reduced the symptoms of collagen-induced arthritis (CIA) $[27,28]$. ZSTK474, a pan-class I PI3K inhibitor, was also found to improve inflammation and disease progression in RA animal models [29]. However, few studies focused on the application of PI3K/AKT inhibitors on the OA treatments. Cartilage erosion in the collagenase-induced osteoarthritis (CIOA) model was ultimately driven by the remodeled synovial lining and direct effect of synoviocytes. In the CIOA mice, we demonstrated that the collagenase injection resulted in osteoarthritis-like changes including higher degree of synovitis, more pronounced chondral defects occurring in the medial tibio-femoral compartment, and not completely mineralized osteophytes. Our results showed that there was no significant effect on synovitis score and cartilage damage score in CIOA mice injected i.p. with PI3K inhibitor, LY294002. Li et al. injected LY294002 with $20 \mathrm{mg} / \mathrm{kg}$ intraperitoneally for the treatment of rheumatoid arthritis in rats. After 3 weeks, the symptoms were relieved and synovial hyperplasia and inflammatory cell infiltration improved [30]. Although PI3K inhibitors have great efficacy, studies have shown that they may be at the expense of increased toxicity. Hu et al. also reported weight loss and dry skin in mice with ovarian cancer after intraperitoneal injection of LY294002 at 100 $\mathrm{mg} / \mathrm{kg}$ [31]. Extensive inhibition of PI3K is likely to cause significant toxicity because PI3K I is critical for normal physiological processes such as glucose homeostasis and immune responses. Therefore, based on the consideration that LY294002 toxicity may cause death in mice, we prudently selected $50 \mathrm{mg} / \mathrm{kg}$ as the final therapeutic dose. However, the difference in pathogenesis and characteristics between the carcinoma and OA imply the distinct dose and duration of therapy of PI3K inhibitors, which needs further research.

We already know that for most of the in vivo processes involved, the effects of class I PI3K inhibition are usually partial. Specifically, the inhibition of PI3KY can be blunt recruitment and activation of innate immune cells, which is not complete; the inhibition of PI3K $\delta$ prevents a normal antibody response, but some other antibodies are made; inhibition of PI3K $\delta$ and $\beta$ can inhibit antibody-dependent activation of neutrophils and macrophages, but bacterial uptake and killing are relatively unscathed. On the other hand, macrophages are important constituent cells of the synovial lining layer. In addition to regulation of synovial inflammation, macrophages can also mediate the process of cartilage damage.
After clearance of macrophages in CIOA synovial inflammation, cartilage destruction was significantly alleviated. Synovitis and cartilage destruction in the CIOA model of S100A $9^{-/-}$mice were significantly improved [32] Although we confirmed that LY294002 can inhibit the ability of OA FLS invasion, its role on macrophages is not clear. It may explain that LY294002 did not improve synovitis and cartilage destruction in the CIOA model. In addition, since multiple signaling pathways that regulate OA FLS are independent of each other and balance with each other, a single block of a pathway may not be a good inhibitor of hyperplasia and activation of OA FLS, resulting in less than ideal results. Thus, the robust and redundant processes that underlie the inflammatory response may allow an opportunity to inhibit class I PI3Kdependent processes to a level in which a significant decrease of Cadherin-11 expression is possible but alleviation of the pathology is not complete. Furthermore, the inflammatory cytokines in the OA pathological process trigger a variety of downstream signaling pathway.

The protective effect of anti-Cadherin-11 therapy has been demonstrated convincingly in a range of arthritis models. Lee et.al [29] found that anti-Cadherin-11 displays moderate amelioration of established $\mathrm{K} / \mathrm{BxN}$ serum transfer arthritis. Kou et al. [33] indicated that blocking Cadherin-11 partially reversed the TMJ inflammatory pain and estradiol-potentiated proliferation of synovial lining cells. This study was the first to investigate the anti-Cadherin-11 therapeutics in the CIOA mice, which mostly resembled the synovium inflammation and cartilage damage in the knee OA patients. In the study, not only the synovitis was partially attenuated after the Cadherin-11 antibody injection, but also the condition of cartilage damage was improved to some extent, especially in the MTP and MFC. Overexpression of Cadherin-11 in the synoviocytes might be positively related to the increased invasive capacity of FLS and articular cartilage destruction while anti-Cadherin-11 antibody significantly alleviates the pathology changes.

There were some limitations of our study. The role of other cytokines, such as IL-1 and IL-6, on activating OA FLS through the PI3K/Akt signaling pathway has not been investigated. Moreover, CIOA mice models are not the same as human OA, and the applicability of lowtoxicity PI3K/Akt inhibitor or Cadherin-11-targeted therapy to other OA models and human OA still needs to be evaluated.

\section{Conclusions}

PI3K/Akt pathway was associated with TNF- $\alpha$-induced activation of fibroblast-like synoviocytes in OA, which may be involved in the pathogenesis of osteoarthritis. Anti-Cadherin-11 therapy in CIOA mice could attenuate the pathological changes of OA joints, although PI3K/ 
AKT inhibitor's influence was not complete. Further clarification of the mechanism underlying the upregulation of PI3K/Akt induced by TNF- $\alpha$ should be examined with promising results in the field of potential alternative treatments of OA. More detailed studies should provide additional insights into the mechanisms relevant to the development and progression of osteoarthritis.

\section{Abbreviations}

ANOVA: Analysis of variance; cDNA: Complementary DNA; CIOA: Collagenaseinduced osteoarthritis; DMEM: Dulbecco's modified Eagle's medium; FBS: Fetal bovine serum; FLS: Fibroblast-like synoviocytes; GAPDH: Glyceraldehyde-3-phosphate dehydrogenase; GSK-3ß: Glycogen synthase kinase 3 beta; MMP: Matrix metalloproteinase; OA: Osteoarthritis; PBS: Phosphate-buffered saline; PI3K: Phosphatidylinositol 3-kinase; qRTPCR: Quantitative real-time polymerase chain reaction; RA: Rheumatoid arthritis; SD: Standard deviation; SEM: Standard error of the mean; TNFa: Tumor necrosis factor a

\section{Acknowledgements}

We thank Tingting Wang and Shan Wang at the Institute of Clinical Molecular Biology and Central Laboratory for experimental equipment and technical guidance.

\section{Authors' contributions}

SYL, CXC, and TZS designed the study, performed the statistical analysis, and prepared the manuscript. TZS supervised the experiments and helped in reviewing the manuscript. All the authors revised and approved the final manuscript. SYL and CXC contributed equally to this study.

\section{Funding}

The research was funded by the National Nature Science Funds from China (81472123) and Peking University Medicine Seed Fund for Interdisciplinary Research (BMU2018MX011), supported by "the Fundamental Research Funds for the Central Universities.

\section{Availability of data and materials}

The datasets used and/or analyzed during the current study are available from the corresponding author on reasonable request.

\section{Ethics approval and consent to participate}

Written informed consent was obtained from all patients, and the research protocol was approved by the Institutional Review Board of the People's Hospital, Peking University (Beijing, China). Animal experimental protocols were approved by Peking University Institutional Animal Care and Use Committee.

\section{Consent for publication}

Informed consent was obtained from all individual participants included in the study. All patients provided written informed consent for the use of chondrocytes.

\section{Competing interests}

The authors declare that they have no competing interests.

\section{Author details}

'Arthritis Clinic and Research Center, People's Hospital, Peking University, Beijing 100044, People's Republic of China. Institute of Sports Medicine, Peking University Third Hospital, Peking University, Beijing 100044, People's Republic of China. ${ }^{3}$ The Institute of Clinical molecular Biology and the Central Lab, Peking University, People's Hospital, Peking University, Beijing 100044, People's Republic of China.
Received: 3 June 2019 Accepted: 25 September 2019

Published online: 11 December 2019

\section{References}

1. Saxne T, Glennås A, Kvien T, Melby K, Heinegård D. Release of cartilage macromolecules into the synovial fluid in patients with acute and prolonged phases of reactive arthritis. Arthritis Rheum. 1993;36(1):20-5.

2. Veale DJ. The role of arthroscopy in early arthritis. Clin Exp Rheumatol. 1999; 17(1):37-8.

3. Felson DT, McLaughlin S, Goggins J, LaValley MP, Gale ME, Totterman S, et al. Bone marrow edema and its relation to progression of knee osteoarthritis. Ann Intern Med. 2003;139(5 Pt 1):330-6.

4. Sellam J, Berenbaum F. The role of synovitis in pathophysiology and clinical symptoms of osteoarthritis. Nat Rev Rheumatol. 2010;6(11):625-35.

5. Ayral X, Pickering EH, Woodworth TG, Mackillop N, Dougados M. Synovitis: a potential predictive factor of structural progression of medial tibiofemoral knee osteoarthritis -- results of a 1 year longitudinal arthroscopic study in 422 patients. Osteoarthr Cartil. 2005;13(5):361-7.

6. Guermazi A, Roemer F, Hayashi D, Crema M, Niu J, Zhang Y, et al. Assessment of synovitis with contrast-enhanced MRI using a whole-joint semiquantitative scoring system in people with, or at high risk of, knee osteoarthritis: the MOST study. Ann Rheum Dis. 2011;70(5):805-11.

7. de Lange-Brokaar B, loan-Facsinay A, van Osch G, Zuurmond A, Schoones J, Toes $R$, et al. Synovial inflammation, immune cells and their cytokines in osteoarthritis: a review. Osteoarthr Cartil. 2012;20(12):1484-99.

8. Roemer FW, Guermazi A, Hunter DJ, Niu J, Zhang Y, Englund M, et al. The association of meniscal damage with joint effusion in persons without radiographic osteoarthritis: the Framingham and MOST osteoarthritis studies. Osteoarthr Cartil. 2009;17(6):748-53.

9. Kiener HP, Brenner MB. Building the synovium: cadherin-11 mediates fibroblast-like synoviocyte cell-to-cell adhesion. Arthritis Res Ther. 2005; 7(2):49-54.

10. Kiener HP, Lee DM, Agarwal SK, Brenner MB. Cadherin-11 induces rheumatoid arthritis fibroblast-like synoviocytes to form lining layers in vitro. Am J Pathol. 2006;168(5):1486-99.

11. Chang SK, Gu Z, Brenner MB. Fibroblast-like synoviocytes in inflammatory arthritis pathology: the emerging role of cadherin-11. Immunol Rev. 2010; 233(1):256-66

12. Altman R, Asch E, Bloch D, Bole G, Borenstein D, Brandt $K$, et al. Development of criteria for the classification and reporting of osteoarthritis. Classification of osteoarthritis of the knee. Diagnostic and therapeutic criteria Committee of the American Rheumatism Association. Arthritis Rheum. 1986;29(8):1039-49.

13. Ding $X$, Zhang $Y$, Huang $Y$, Liu S, Lu H, Sun T. Cadherin-11 involves in synovitis and increases the migratory and invasive capacity of fibroblast-like synoviocytes of osteoarthritis. Int Immunopharmacol. 2015;26(1):153-61.

14. Chang SK, Noss EH, Chen M, Gu Z, Townsend K, Grenha R, et al. Cadherin-11 regulates fibroblast inflammation. Proc Natl Acad Sci U S A. 2011;108(20):8402-7.

15. Vandooren B, Cantaert T, ter Borg M, Noordenbos T, Kuhlman R, Gerlag D, et al. Tumor necrosis factor alpha drives cadherin 11 expression in rheumatoid inflammation. Arthritis Rheum. 2008:58(10):3051-62.

16. van der Kraan PM, Vitters EL, van Beuningen $H M$, van de Putte $L B$, van den Berg WB. Degenerative knee joint lesions in mice after a single intraarticular collagenase injection. A new model of osteoarthritis. J Exp Pathol (Oxford). 1990;71(1):19-31.

17. Chambers MG, Bayliss MT, Mason RM. Chondrocyte cytokine and growth factor expression in murine osteoarthritis. Osteoarthr Cartil. 1997;5(5):301-8.

18. Cho H, Chung J, Song K, Noh K, Kim B, Chung E, et al. Apoptosis inhibitor-5 overexpression is associated with tumor progression and poor prognosis in patients with cervical cancer. BMC Cancer. 2014;14:545.

19. Feldmann M. Pathogenesis of arthritis: recent research progress. Nat Immunol. 2001;2(9):771-3.

20. Zhang $H G$, Wang $Y$, Xie JF, Liang $X$, Liu $D$, Yang $P$, et al. Regulation of tumor necrosis factor alpha-mediated apoptosis of rheumatoid arthritis synovial fibroblasts by the protein kinase Akt. Arthritis Rheum. 2001:44(7):1555-67.

21. Hay N. The Akt-mTOR tango and its relevance to cancer. Cancer Cell. 2005; 8(3):179-83.

22. Bartok B, Hammaker D, Firestein G. Phosphoinositide 3-kinase $\delta$ regulates migration and invasion of synoviocytes in rheumatoid arthritis. J Immunol. 2014;192(5):2063-70. 
23. Lin S, Fan W, Sun L, Li F, Zhao R, Zhang L, et al. The saponin DT-13 inhibits gastric cancer cell migration through down-regulation of CCR5-CCL5 axis. Chin J Nat Med. 2014;12(11):833-40.

24. Berod L, Heinemann C, Heink S, Escher A, Stadelmann C, Drube S, et al. PI3Kgamma deficiency delays the onset of experimental autoimmune encephalomyelitis and ameliorates its clinical outcome. Eur J Immunol. 2011;41(3):833-44.

25. Mengshol JA, Vincenti MP, Brinckerhoff CE. IL-1 induces collagenase-3 (MMP-13) promoter activity in stably transfected chondrocytic cells: requirement for Runx-2 and activation by P38 MAPK and JNK pathways. Nucleic Acids Res. 2001;29(21):4361-72. PMID:11691923.

26. Montaseri A, Busch F, Mobasheri A, Buhrmann C, Aldinger C, Rad JS, Shakibaei M. IGF-1 and PDGF-bb suppress IL-1 $\beta$-induced cartilage degradation through down-regulation of NF-KB signaling: involvement of Src/PI-3K/AKT pathway. PLoS One. 2011;6(12):e28663. https://doi.org/10. 1371/journal.pone.0028663. Epub 2011 Dec 14. PMID: 22194879.

27. Oka Y, Yabuuchi T, Oi T, Kuroda S, Fujii Y, Ohtake H, et al. Discovery of N-\{5[3-(3-hydroxypiperidin-1-yl)-1,2,4-oxadiazol-5-yl]-4-methyl-1,3-thiazol-2-yl \}acetamide (TASP0415914) as an orally potent phosphoinositide 3-kinase gamma inhibitor for the treatment of inflammatory diseases. Bioorg Med Chem. 2013;21(24):7578-83.

28. Haruta K, Mori S, Tamura N, Sasaki A, Nagamine M, Yaguchi S, et al. Inhibitory effects of ZSTK474, a phosphatidylinositol 3-kinase inhibitor, on adjuvant-induced arthritis in rats. Inflamm Res. 2012:61(6):551-62.

29. Lee DM, Kiener HP, Agarwal SK, Noss EH, Watts GF, Chisaka O, et al. Cadherin-11 in synovial lining formation and pathology in arthritis. Science. 2007;315(5814):1006-10.

30. Li GQ, Zhang Y, Liu D, Qian YY, Zhang H, Guo SY, et al. PI3 kinase/Akt/HIFlalpha pathway is associated with hypoxia-induced epithelial-mesenchymal transition in fibroblast-like synoviocytes of rheumatoid arthritis. Mol Cell Biochem. 2013;372(1-2):221-31.

31. Hu L, Zaloudek C, Mills GB, Gray J, Jaffe RB. In vivo and in vitro ovarian carcinoma growth inhibition by a phosphatidylinositol 3-kinase inhibitor (LY294002). Clin Cancer Res. 2000;6(3):880-6.

32. Schelbergen RF, Geven EJ, van den Bosch MH, Eriksson $H$, Leanderson $T$, Vogl T, et al. Prophylactic treatment with S100A9 inhibitor paquinimod reduces pathology in experimental collagenase-induced osteoarthritis. Ann Rheum Dis. 2015;74(12):2254-8.

33. Kou XX, Wang XD, Li CS, Bi RY, Meng Z, Li B, et al. Estradiol-potentiated cadherin-11 in synovial membrane involves in temporomandibular joint inflammation in rats. J Steroid Biochem Mol Biol. 2014;143:444-50.

\section{Publisher's Note}

Springer Nature remains neutral with regard to jurisdictional claims in published maps and institutional affiliations.

Ready to submit your research? Choose BMC and benefit from:

- fast, convenient online submission

- thorough peer review by experienced researchers in your field

- rapid publication on acceptance

- support for research data, including large and complex data types

- gold Open Access which fosters wider collaboration and increased citations

- maximum visibility for your research: over $100 \mathrm{M}$ website views per year

At $\mathrm{BMC}$, research is always in progress.

Learn more biomedcentral.com/submissions 